\title{
Role of axillary ultrasound in the preoperative diagnosis of lymph node metastases in patients affected by breast carcinoma
}

\section{Ruolo dell'ecografia del cavo ascellare nella diagnosi pre-operatoria di metastasi linfonodali in pazienti affette da carcinoma mammario}

\author{
A. Luparia • P. Campanino • R. Cotti • D. Lucarelli • M. Durando • G. Mariscotti • G. Gandini \\ Istituto di Radiologia Diagnostica ed Interventistica, Università di Torino, ASO San Giovanni Battista di Torino, Sede Molinette, \\ Via Genova 3, 10126 Torino \\ Correspondence to: A. Luparia, Tel.: +39-011-6336622, Fax: +39-011-6960310, e-mail: andrea.luparia@ hotmail.it
}

Received: 23 December 2008 / Accepted: 3 April 2009 / Published online: 30 September 2009

C) Springer-Verlag 2009

\begin{abstract}
Purpose. This study was conducted to evaluate the diagnostic accuracy of axillary ultrasound (US) alone or in combination with fine-needle-aspiration cytology (FNAC) in patients with breast carcinoma, in comparison with the final histological examination (sentinel node biopsy and/or axillary dissection).

Materials and methods. Between January 2005 and June 2008, we evaluated 427 breast cancer patients with axillary US. The findings were classified according to the following criteria: hilum and cortex morphology, ratio between longitudinal and transverse diameter and ratio between hilar and longitudinal diameter of the lymph node. Patients with breast lesions $\leq 3 \mathrm{~cm}(\mathrm{n}=147)$ underwent FNAC of the most suspicious lymph node.

Results. Overall concordance between axillary US and final histological examination was $85 \%$, sensitivity was $72.3 \%$ and specificity was $93.4 \%$. Concordance between FNAC and final histological examination was 93\%, sensitivity was $88.1 \%$ and specificity was $100 \%$. Conclusions. In $48.3 \%$ of patients, the combination of axillary US and FNAC guided treatment decisions towards immediate axillary dissection, thus sparing the patients sentinel node biopsy, with a significant reduction of costs and hospitalization time.
\end{abstract}

Keywords Breast carcinoma $\cdot$ Axillary space $\cdot$ Lymph nodes $\cdot$ Ultrasound

\section{Riassunto}

Obiettivo. Scopo del nostro lavoro è stato valutare, in pazienti affette da carcinoma mammario, l'accuratezza diagnostica dell'ecografia (US) del cavo ascellare da sola oppure associata ad agoaspirato con ago sottile (FNAC) assumendo come parametro di confronto l'esame istologico definitivo (biopsia del linfonodo sentinella elo dissezione ascellare).

Materiali e metodi. Tra gennaio 2005 e giugno 2008 in 427 pazienti affette da neoplasia mammaria è stato eseguito lo studio ecografico del cavo ascellare. Per la classificazione dei reperti ecografici sono stati valutati: la morfologia dell'ilo e della corticale, il rapporto tra diametro longitudinale e trasversale e il rapporto tra diametro della regione ilare e longitudinale del linfonodo. In 147 pazienti, con neoplasia mammaria $\leq 3 \mathrm{~cm}$, è stata eseguita l'US-FNAC del linfonodo più sospetto.

Risultati. Si è evidenziata una concordanza complessiva tra US del cavo ascellare e istologico definitivo dell'85\%, una sensibilità del 72,3\% ed una specificità del 93,4\%. La concordanza dell'US-FNAC con l'istologico definitivo è risultata del 93\%, la sensibilità dell'88,1\% e la specificità del $100 \%$.

Conclusioni. L'ecografia del cavo ascellare associata alla FNAC nel 48,3\% dei casi ha permesso di evitare la biopsia del linfonodo sentinella, orientando il trattamento chirurgico verso una dissezione ascellare immediata, con sensibile riduzione dei costi e del tempo di ospedalizzazione.

Parole chiave Carcinoma mammario - Cavo ascellare . Linfonodi $\cdot$ Ecografia 


\section{Introduction}

Breast cancer prognosis is heavily dependent on the presence or absence of lymph node metastases at presentation [1]. Axillary US may help improve preoperative staging and treatment planning, thereby ensuring better patient compliance and reducing hospitalisation time and costs. The study of axillary lymph nodes is aimed at detecting metastases and avoiding two-step axillary surgery by guiding treatment towards immediate axillary dissection - that is, not preceded by sentinel node biopsy - in the case of positive findings.

In recent years, numerous studies have assessed the role of US in the study of the axilla in an attempt to establish pathognomonic morphostructural criteria that could distinguish metastatic nodes from reactive ones (reactive hyperplasia, inflammation) [1-26]. To correctly stage axillary lymph nodes, US has been combined with US-guided fineneedle-aspiration cytology (FNAC) [1, 2, 14, 16-23] or core biopsy, although the latter, which is more invasive than FNAC, is not widely used [7, 8, 15, 24-26].

The purpose of this study was to evaluate the diagnostic accuracy of axillary US and the adjunctive role of nodal FNAC in the diagnostic workup of patients with breast carcinoma in comparison with final histology of axillary lymph nodes (sentinel node biopsy and/or axillary dissection).

\section{Materials and methods}

Between January 2005 and June 2008, 427 patients with breast carcinoma scheduled for surgical excision of a primary cancer and axillary surgery (sentinel node biopsy and/or axillary dissection) were studied with axillary US. US scans were carried out with dedicated equipment (Technos $700 \mathrm{C}$ or My Lab 25 CV, Esaote, Genoa, Italy) and a 7.5- to $12-\mathrm{MHz}$ probe; all US studies were supplemented by colour and power Doppler imaging. US findings were classified on the basis of commonly reported criteria [1-9]: hilar and cortical morphology, ratio between longitudinal (L) and transverse (T) diameter and ratio between hilar (H) and $\mathrm{L}$ diameter of lymph nodes. Lymph nodes with an $\mathrm{L} / \mathrm{T} \geq 2, \mathrm{H} / \mathrm{L} \geq 50 \%$ and cortical thickness $\leq 2 \mathrm{~mm}$ were considered normal. The US findings were classified into four categories: normal, probably benign (indeterminate), suspicious for malignancy or probably malignant (Table 1). A US finding of any nodal appearance other than those classified as normal constituted the indication for FNAC, which was performed with a 22-gauge needle connected to a standard 20-cc syringe.

All patients provided informed consent before undergoing the sampling procedure. FNAC results were classi-

\section{Introduzione}

La prognosi, in pazienti affette da carcinoma mammario, è fortemente condizionata dalla presenza o meno di metastasi linfonodali al momento della diagnosi [1]. Lo studio ecografico del cavo ascellare, metodica non invasiva, può contribuire ad una corretta stadiazione pre-operatoria $e$ quindi ad una migliore pianificazione terapeutica, assicurando una migliore compliance della paziente e una riduzione dei tempi e dei costi di gestione. Infatti, l'obiettivo dello studio linfonodale è la ricerca dell'eventuale presenza di metastasi, al fine di evitare la chirurgia del cavo ascellare in due tempi, orientando, in caso di riscontro di positività, verso una dissezione ascellare immediata, ciò̀ non preceduta da biopsia del linfonodo sentinella (LS).

Negli ultimi anni, numerosi lavori hanno valutato il ruolo dell'ecografia nello studio del cavo ascellare, cercando di stabilire dei criteri morfostrutturali patognomonici di compromissione metastatica dei linfonodi, al fine di differenziarli da quelli reattivi (iperplasia reattiva, flogosi) [1-26]. Per la corretta stadiazione dei linfonodi ascellari, sono stati inoltre associati all'esame ecografico l'utilizzo dell'agoaspirato linfonodale ecoguidato con ago sottile (FNAC) [1, 2, 14, 16-23] o la core biopsy. Quest'ultima metodica, più invasiva rispetto alla FNAC, non è ancora utilizzata su larga scala [7, 8, 15, 24-26].

Scopo del presente lavoro è stato quello di valutare, in pazienti affette da neoplasia mammaria, sia l'accuratezza diagnostica dell'ecografia del cavo ascellare, sia il ruolo aggiuntivo che il prelievo linfonodale mediante FNAC svolge nel percorso diagnostico, assumendo come parametro di confronto l'esame istologico definitivo dei linfonodi ascellari (biopsia del linfonodo sentinella elo dissezione ascellare).

\section{Materiali e metodi}

Nel periodo tra gennaio 2005 e giugno 2008 è stato eseguito lo studio ecografico del cavo ascellare in 427 pazienti affette da neoplasia mammaria e candidate ad intervento chirurgico di exeresi della neoplasia primitiva e a chirurgia del cavo ascellare (biopsia del linfonodo sentinella elo dissezione ascellare). L'esame ultrasonografico è stato eseguito in tutte le pazienti con apparecchiatura dedicata (Technos 700 C oppure My Lab 25 CV, Esaote, Genova, Italia) con sonda da 7,5-12 MHz e completato con studio color/power doppler. Per la classificazione dei reperti ecografici sono stati utilizzati $i$ parametri più frequentemente riportati in letteratura [1-9]: la morfologia dell'ilo e della corticale, il rapporto tra diametro longitudinale $(L)$ e trasversale $(T)$ e il rapporto tra il diametro della regione ilare $(H)$ e L del linfonodo. Sono stati considerati 
Table 1 Ultrasound (US) classification of axillary lymph nodes

\begin{tabular}{lllll}
\hline US parameters & Normal & Probably benign & Suspicious for malignancy & Probably malignant \\
\hline L/T ratio & $\geq 2$ & $\geq 2$ & $<2$ & $<2$ \\
H/L ratio & $\geq 50 \%$ & $\geq 50 \%$ & $<50 \%$ & $<50 \%$ \\
Cortical thickness $(\mathrm{mm})$ & $\leq 2$ & $>2$ & $>2$ & $>2$ \\
Morphology & Oval shape & Oval shape & Rounder shape & Globular shape \\
Hilum & Clearly visible & Clearly visible & Not completely visible & Absent \\
\hline
\end{tabular}

$L / T$, longitudinal and transverse ratio; $H / L$, hilar and longitudinal ratio

Tabella 1 Classificazione ecografica dei linfonodi

\begin{tabular}{lllll}
\hline Parametri ecografici & Normale & Verosimilmente benigno & Sospetto per malignità & Verosimilmente maligno \\
\hline Rapporto $L / T$ & $\geq 2$ & $\geq 2$ & $<2$ & $<2$ \\
Rapporto $H / L$ & $\geq 50 \%$ & $\geq 50 \%$ & $<50 \%$ & $<50 \%$ \\
Spessore corticale $(\mathrm{mm})$ & $\leq 2$ & $>2$ & $>2$ & $>2$ \\
Morfologia & ovalare & ovalare & rotondeggiante & globosa \\
Ilo & ben visibile & ben visibile & poco visibile & assente \\
\hline
\end{tabular}

$L / T$, diametro linfonodale longitudinale e trasversale; $H / L$, diametro della regione ilare longitudinale linfonodale

fied in accordance with the European Guidelines [27] into five categories: $\mathrm{C} 1=$ inadequate, $\mathrm{C} 2=$ negative, $\mathrm{C} 3=$ equivocal probably benign, $\mathrm{C} 4=$ suspicious of malignancy, C5=malignant. Adequacy of cytological specimens was evaluated extemporaneously (slide smearing, methanol fixation and haematoxylin-eosin staining) by an on-site pathologist. All patients with a breast mass $>3 \mathrm{~cm}$ or with positive lymph node cytology proceeded directly to axillary dissection; patients with a mass $\leq 3 \mathrm{~cm}$ with negative or equivocal cytology were referred for sentinel node biopsy after localisation with radioactive tracer (Tc-99-m sestamibi) or vital dye (Patent blue in harmless doses) injection into the peritumoural area.

Diagnostic accuracy, sensitivity, specificity, positive predictive value (PPV) and negative predictive value (NPV) of axillary US and US-guided FNAC in assessing the presence of nodal metastases were established comparing the results with final histology of sentinel node biopsy and/or axillary dissection. Statistical assessment of FNAC accuracy was performed by excluding inadequate samples (C1) from the calculations and considering $\mathrm{C} 2-\mathrm{C} 3$ findings as negative and $\mathrm{C} 4-\mathrm{C} 5$ as positive.

\section{Results}

Histological characterisation of breast carcinomas

Of the 427 patients (aged 26-81 years, mean age 60.9 years), 401 (93.9\%) were affected by infiltrating breast carcinoma with a mean diameter of $1.88 \mathrm{~cm}$ [standard deviation $(\mathrm{SD}) \pm 1.2 \mathrm{~cm})$ ], whereas $26(6.1 \%)$ had carcinoma in situ (Tis) [mean diameter $2.77 \mathrm{~cm}(\mathrm{SD} \pm 1.28 \mathrm{~cm})$ ]. All normali valori di $L / T \geq 2$, di $H / L \geq 50 \%$ e di spessore della corticale $\leq 2 \mathrm{~mm}$. Sulla base di questi parametri, abbiamo classificato $i$ reperti in quattro categorie: linfonodo normale, verosimilmente benigno (indeterminato), sospetto per malignità o verosimilmente maligno (Tabella 1). L'indicazione all'utilizzo della FNAC, mediante ago da 22 G collegato a siringa standard da $20 \mathrm{cc}$, è stata la caratterizzazione dei reperti ecografici classificati diversamente dall'aspetto linfonodale normale.

Tutte le pazienti, prima di essere sottoposte a prelievo, hanno firmato un consenso informato. L'esame citologico su agoaspirato ̀̀ stato classificato in 5 categorie: $C 1=$ inadeguato, $C 2=$ negativo, $C 3=$ dubbio (lesione probabilmente benigna), $C 4=$ sospetto di malignità, $C 5=$ positivo, in accordo con le Linee Guida Europee [27]. Il materiale prelevato per l'esame citologico è stato sottoposto ad osservazione estemporanea (striscio su vetrino, fissazione in metanolo e colorazione con ematossilina-eosina) da parte dell'anatomo-patologo per valutarne l'idoneità. Tutte le pazienti con neoplasia mammaria $>3 \mathrm{~cm}$ o con citologia linfonodale positiva sono state sottoposte direttamente a dissezione ascellare; le pazienti con neoplasia $\leq 3 \mathrm{~cm}$ con citologia negativa o dubbia sono state inviate a biopsia del linfonodo sentinella previa localizzazione mediante iniezione nella zona peri-tumorale di un tracciante radioattivo (Tc-99-m sestamibi) o di un colorante vitale (patent blue in dosi assolutamente prive di qualsiasi effetto nocivo).

L'accuratezza diagnostica, la sensibilità, la specificità, il valore predittivo positivo (VPP) e il valore predittivo negativo (VPN) della valutazione ultrasonografica dei linfonodi ascellari e della FNAC ecoguidata per la presenza di metastasi linfonodali sono stati confrontati con i reperti istologici definitivi della biopsia del linfonodo sentinella elo della 
patients underwent surgery: $63.7 \%$ of cancers (272/427) were $\mathrm{T} 1$ (T1a in nine patients; $\mathrm{T} 1 \mathrm{~b}$ in 74 ; $\mathrm{T} 1 \mathrm{c}$ in 189 ), and $27.9 \%(119 / 427)$ were $\mathrm{T} 2$, whereas only a small number of patients had T3-T4 $(2.3 \%, 10 / 427)$ cancers at final histo$\log$.

The most common histological type of invasive tumour was invasive ductal carcinoma (IDC) [71.3\% (286/401)], followed by invasive lobular carcinoma (ILC) in $10.5 \%$ of cases (42/401), mixed ductal-lobular carcinoma in $4.5 \%$ (18/401) and other histological types (apocrine, tubular, mucinous, cribriform, inverted micropapillary, neuroendocrine, medullar carcinoma) in the remaining $13.7 \%$ (55/401). Histological nuclear grading of infiltrating tumours according to Elston and Ellis [28] was low (G1) in $24.2 \%$, intermediate (G2) in $42.8 \%$ and high (G3) in $33 \%$ of cases. The most common histological type of carcinoma in situ was ductal (DCIS) in 21 cases, comedogenic in three, cribriform in one and papillary in one.

Concordance of US, US-guided FNAC and final histological result

Preoperative axillary node US was negative for nodal abnormalities in 203/427 cases and positive for one or more US criteria in 224/427 cases (Table 2). In 203/427 patients with normal US findings ( $\mathrm{L} / \mathrm{T} \geq 2$ and cortical thickness $\leq 2$ $\mathrm{mm}$ ), FNAC was not performed, and final histology (sentinel node biopsy and/or axillary dissection) confirmed the negative US findings in $87.2 \%$ of cases (177/203). In the remaining 26 cases ( $12.8 \%$ ), the final histological examination revealed nodal metastases (in four cases micrometastases $\leq 2 \mathrm{~mm}$ ). Of the 224 patients exhibiting echostructural alterations on axillary US, 147 underwent FNAC to characterise the US findings. Despite abnormal US findings in the dissezione ascellare. Le valutazioni statistiche relative all'accuratezza della FNAC sono state eseguite escludendo dai calcoli i campioni inadeguati (C1) e considerando gli esami citologici su agoaspirato C2-C3 come negativi e C4C5 come positivi.

\section{Risultati}

Caratteristiche istologiche delle neoplasie mammarie

Delle 427 pazienti (età media 60,9 anni, range 26-81 anni), 401 (93,9\%) erano affette da carcinoma mammario infiltrante con un diametro medio di $1,88 \mathrm{~cm}$ (deviazione standard $[S D]=1,2 \mathrm{~cm})$, mentre $26(6,1 \%)$ da carcinoma in situ (Tis) [diametro medio di $2,77 \mathrm{~cm}(S D=1,28 \mathrm{~cm})]$. Tutte le pazienti sono state sottoposte ad intervento chirurgico e all'istologico definitivo il 63,7\% delle neoplasie (272/427) è risultato T1 (Tla in 9 pazienti; Tlb in 74; T1c in 189), il 27,9\% (119/427) T2 e solo un numero ristretto T3-T4 $(2,3 \%, 10 / 427)$.

L'istotipo prevalente nei casi di neoplasia infiltrante è stato il carcinoma duttale infiltrante (CDI) $(71,3 \%$, 286/401), seguito da quello lobulare (CLI) nel 10,5\% dei casi (42/401), dalla forma mista duttale e lobulare nel 4,5\% (18/401) e nel restante 13,7\% (55/401) da altri tipi istologici (apocrino, tubulare, mucinoso, cribriforme, micropapillare invertito, neuroendocrino, midollare). Il grading nucleare istologico dei tumori infiltranti secondo ElstonEllis [28] è risultato: basso-G1 nel 24,2\%, intermedio-G2 nel 42,8\% e alto-G3 nel 33\%. Tra i carcinomi in situ l'istotipo prevalente è risultato essere il carcinoma duttale in situ (CDIS) in 21 casi, comedonico in 3 casi, un cribriforme e un papillare.

Table 2 Concordance between axillary ultrasound (US) and final histological examination

\begin{tabular}{lllll}
\hline US appearance & Total & \multicolumn{2}{c}{ Final histological examination } & Concordance (\%) \\
& & Negative $(\mathrm{N}-)$ & Positive $(\mathrm{N}+)$ & \\
\hline Normal & 203 & 177 & 26 & 87.2 \\
Probably benign & 84 & 63 & 21 & 75 \\
Suspicious for malignancy or probably malignant & 140 & 17 & 123 & 87.9 \\
Total & 427 & 257 & 170 & \\
\hline
\end{tabular}

Tabella 2 Concordanza tra reperti ecografici linfonodali ed istologia definitiva

\begin{tabular}{|c|c|c|c|c|}
\hline Aspetto ecografico & Totale & $\begin{array}{l}\text { Istologico definitivo } \\
\text { Negativo }(N-)\end{array}$ & Positivo $(\mathrm{N}+)$ & Concordanza (\%) \\
\hline Normale & 203 & 177 & 26 & 87,2 \\
\hline Verosimilmente benigno & 84 & 63 & 21 & 75 \\
\hline Sospetto per malignità & 140 & 17 & 123 & 87,9 \\
\hline o verosimilmente maligno & & & & \\
\hline Totale & 427 & 257 & 170 & \\
\hline
\end{tabular}




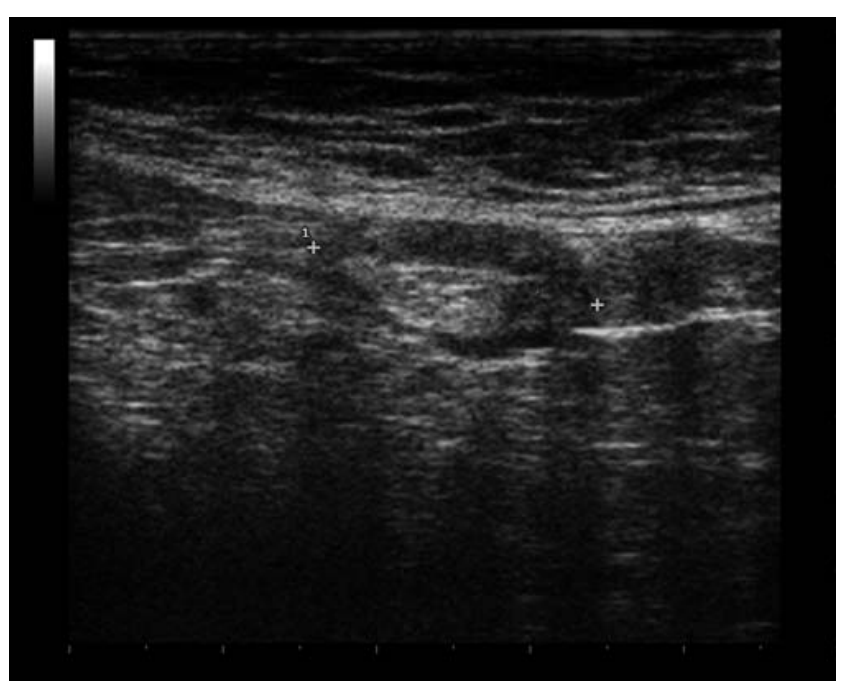

Fig. 1 Oval-shaped lymph node $\mathrm{L} / \mathrm{T}>2$, L diameter $=1.9 \mathrm{~cm}, \mathrm{~T}$ diameter $=0.9 \mathrm{~cm}$; the cortex is thickened $(>2 \mathrm{~mm})$, whereas the $\mathrm{H}$ region is clearly visible and hyperechoic $(\mathrm{H}=1.4 \mathrm{~cm}, \mathrm{H} / \mathrm{L}>50 \%)$. Probably benign. Axillary US-FNAC: lymphocytes (C2). Final histological examination: absence of lymph node metastases from invasive ductal carcinoma (diameter=1 cm) (sentinel node biopsy: 0/1, N-).

Fig. 1 Linfonodo con morfologia ovalare (rapporto $L / T>2$ ), $L=1,9 \mathrm{~cm}$, $T=0,9 \mathrm{~cm}$, con corticale ispessita $(>2 \mathrm{~mm})$ e ilo iperecogeno ben evidente $(H=1,4 \mathrm{~cm}$, rapporto $H / L>50 \%)$. Verosimilmente benigno. US-FNAC linfonodale: linfociti (C2). Istologico definitivo: assenza di metastasi linfonodali di carcinoma duttale infiltrante (diametro $=1 \mathrm{~cm}$ ) (linfonodo sentinella: $0 / 1, N_{-}$).

remaining 77 cases, FNAC was not performed because of the depth of lymph nodes, proximity of large axillary blood vessels or a preoperative diagnosis of a tumour $>3 \mathrm{~cm}$.

Considering US alterations only, among 84/224 lymph nodes classified as probably benign (indeterminate) ( $\mathrm{L} / \mathrm{T} \geq 2$, cortical thickness $>2 \mathrm{~mm}$ ), benignity was confirmed by definitive histological examination in $75 \%$ of cases (63/84) (Fig. 1). Among 55/84 lymph nodes studied with FNAC, 39 were C2, one was C3 and three were C5, resulting in $88.4 \%$ concordance with definitive histology (38/43). In 12/55 cases, cytology was not diagnostic (C1) owing to inadequate sampling. In 140/224 cases in which lymph nodes exhibited echostructural alterations classified as suspicious for malignancy $(\mathrm{L} / \mathrm{T}<2$, cortical thickening $>2 \mathrm{~mm}$, a more rounded shape, partially visible hilum) or probably malignant (L/T $<2$, cortical thickening $>2 \mathrm{~mm}$, globular shape, invisible hilum), the definitive histological examination confirmed the presence of nodal metastases in $87.9 \%$ of cases (Figs 2, 3a, b). FNAC was performed on $92 / 140$ nodes (18 C2, one C4, 67 C5), with a concordance between cytology and final histology of $95.3 \%$ (82/86). In six cases, cytology was not diagnostic (C1) owing to inadequate sampling (Tables 2 and 3).

With regard to the correlation between $\mathrm{L} / \mathrm{T}$ ratio of the lymph node and final histology, out of the 287/427 lymph nodes with $\mathrm{L} / \mathrm{T} \geq 2$ (index of probably benign node), 240

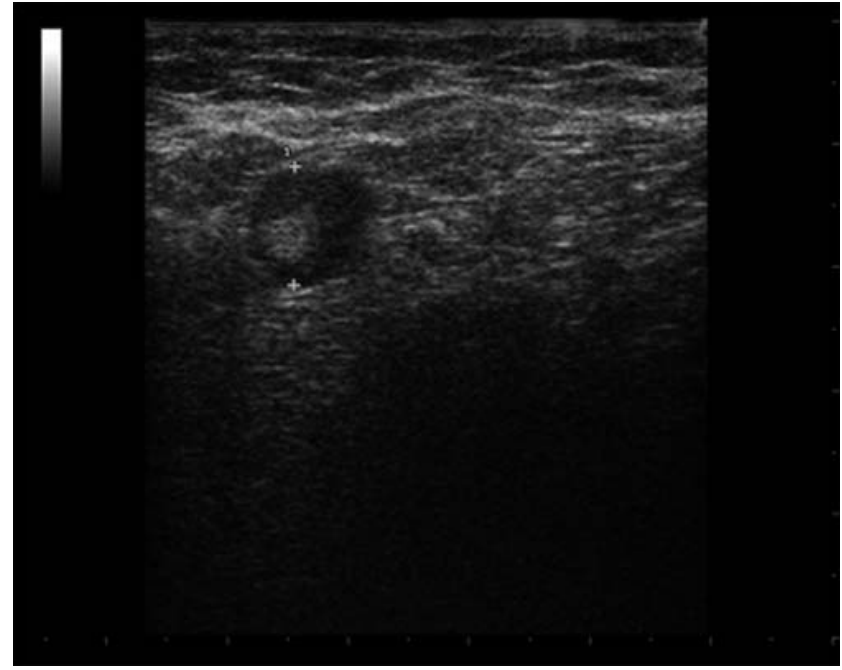

Fig. 2 Lymph node with cortical thickening $(>2 \mathrm{~mm}), \mathrm{L}=1.1 \mathrm{~cm}, \mathrm{~T}=0.8 \mathrm{~cm}$, $\mathrm{H}=0.4 \mathrm{~cm}, \mathrm{~L} / \mathrm{T}<2$ and $\mathrm{H} / \mathrm{L}<50 \%$. Suspicious for malignancy. Axillary US-FNAC: tumour cells (C5). Final histological examination: presence of nodal metastases from invasive ductal carcinoma (diameter $=1.5 \mathrm{~cm})($ axillary dissection: $3 / 23, \mathrm{~N}+$ ).

Fig. 2 Linfonodo con corticale ispessita ( $>2 \mathrm{~mm}), L=1,1 \mathrm{~cm}, T=0,8 \mathrm{~cm}$, $H=0,4 \mathrm{~cm}$, rapporto $L / T<2$ e rapporto $H / L<50 \%$. Sospetto per malignità. US-FNAC linfonodale: cellule tumorali (C5). Istologico definitivo: presenza di metastasi linfonodali di carcinoma duttale infiltrante (diametro=1,5 cm) (dissezione ascellare: $3 / 23, \mathrm{~N}+$ ).

Concordanza tra ecografia, FNAC ecoguidata e risultato istologico definitivo

L'esame ecografico del cavo ascellare eseguito prima dell'intervento chirurgico è risultato negativo per alterazioni linfonodali in 203/427 casi e positivo per alterazioni di uno o più parametri ecografici in 224/427 casi (Tabella 2). Nelle 203/427 pazienti con reperto ecografico normale (con rapporto $L / T \geq 2$ e spessore della corticale $\leq 2 \mathrm{~mm}$ ) non è stata eseguita FNAC: l'esame istologico definitivo (LS elo dissezione ascellare) ha confermato la negatività ecografica nell'87,2\% dei casi (177/203). Nei restanti 26 casi (12,8\%) all'istologico definitivo è stata riscontrata la presenza di metastasi linfonodali (in 4 casi micrometastasi $2 \mathrm{~mm}$ ). Delle 224 pazienti che presentavano all'esame ultrasonografico del cavo ascellare alterazioni ecostrutturali, in 147 è stata eseguita FNAC per la caratterizzazione dei reperti ecografici. Negli altri 77/224 casi, nonostante il sospetto ecografico, non è stata eseguita FNAC per la sede profonda dei linfonodi, per la vicinanza di grossi vasi sanguigni dell'ascella o per la diagnosi pre-operatoria di neoplasia di dimensioni superiori ai $3 \mathrm{~cm}$.

Considerando esclusivamente le alterazioni ecografiche, in 84/224 casi classificati come linfonodi verosimilmente benigni (indeterminati) $(L / T \geq 2$, spessore della corticale $>2$ 

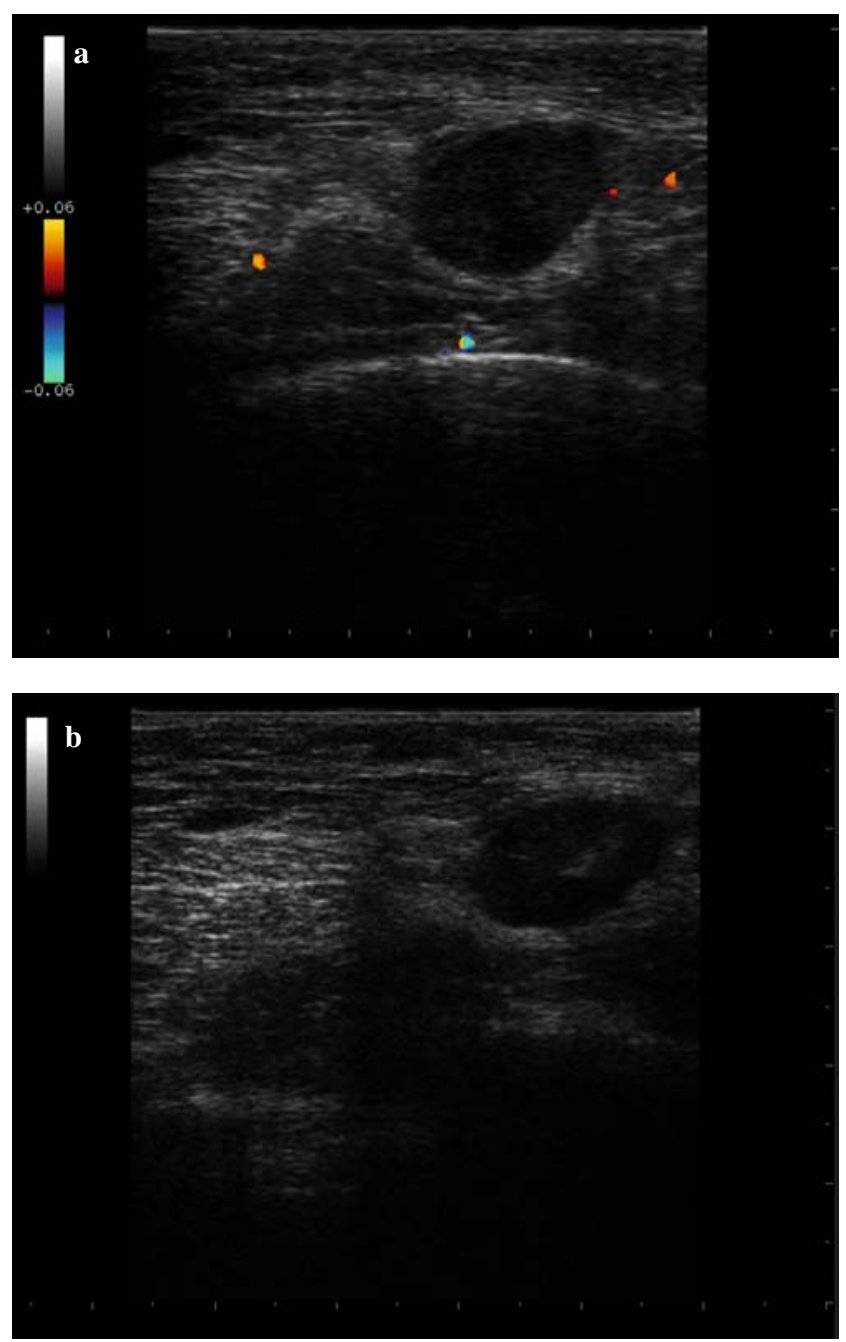

Fig. 3a Lymph node with irregular echostructure: $\mathrm{L}=1.3 \mathrm{~cm}, \mathrm{~T}=1.2 \mathrm{~cm}$, rounded shape $(\mathrm{L} / \mathrm{T}=1)$, and no clearly visible hilum. Probably malignant. b Axillary US-FNAC: tumour cells (C5). Final histological examination: presence of lymph node metastases from invasive ductal carcinoma (diameter $=2.6 \mathrm{~cm})$ (axillary dissection: $4 / 18, \mathrm{~N}+$ ).

Fig. 3a Linfonodo ad ecostruttura sovvertita: $L=1,3 \mathrm{~cm}, T=1,2 \mathrm{~cm}$, forma rotondeggiante (rapporto $L / T=1$ ) e ilo non evidente. Verosimilmente maligno. $\boldsymbol{b}$ US-FNAC linfonodale con ago da $22 \mathrm{G}$ : cellule tumorali (C5). Istologico definitivo: presenza di metastasi linfonodali di carcinoma duttale infiltrante (diametro $=2,6 \mathrm{~cm}$ ) (dissezione ascellare: $4 / 18, \mathrm{~N}+$ ).

were found to be free of metastasis on axillary surgery, with a specificity of $83.6 \%$ (240/287). Out of 140/427 lymph nodes with $\mathrm{L} / \mathrm{T}<2$ (index of probably malignant node), final histology confirmed the presence of metastases in 123/140 cases, with a sensitivity of $87.9 \%$ (Tables 4 and 5).

Compared with final histological examination, axillary US had $85 \%$ diagnostic accuracy (363/427), 72.3\% sensitivity (27.7 \% false negatives) and $93.4 \%$ specificity. Compared with final histology, axillary FNAC had 93\% diagnostic accuracy (120/129), 88.8\% sensitivity and 100\% specificity (values calculated excluding the $\mathrm{C} 1$ ) (Table 6). mm) si è avuta la conferma di benignità all'istologico definitivo linfonodale nel 75\% (63/84) dei casi (Fig. 1). Nei $55 / 84$ casi in cui è stata eseguita FNAC si sono ottenuti 39 C2, 1 C3, 3 C5, con una concordanza con l'istologia definitiva pari all'88,4\% (38/43). In 12/55 la citologia non è risultata diagnostica $(\mathrm{Cl})$ per la scarsità del materiale prelevato. Nei 140/224 casi in cui i linfonodi presentavano alterazioni ecostrutturali sospette per malignità $(L / T<2$, ispessimento della corticale $>2 \mathrm{~mm}$, forma più rotondeggiante, ilo parzialmente riconoscibile) o verosimilmente maligne ( $L / T<2$, ispessimento della corticale $>2 \mathrm{~mm}$, forma globosa, ilo asssente) l'istologico definitivo ha confermato nell'87,9\% dei casi la presenza di metastasi linfonodali (Figg. 2 e 3). Tra questi, 92/140 sono stati sottoposti a FNAC (di cui 18 C2, 1 C4 e 67 C5) con una concordanza tra citologia e istologia definitiva del 95,3\% (82/86). In 6 casi la citologia è risultata non diagnostica (C1) per l'esiguità del materiale prelevato (Tabelle 2 e 3).

Considerando la correlazione tra il rapporto $L / T$ del linfonodo e l'esame istologico definitivo, dei 287/427 linfonodi esaminati con un rapporto $L / T \geq 2$ (indice di probabile benignità linfonodale) 240 sono risultati indenni da metastasi alla chirurgia del cavo ascellare, con una specificità dell'83,6\% (240/287). Dei 140/427 linfonodi con un rapporto $L / T<2$ (indice di probabile malignità linfonodale) l'istologico definitivo ha confermato la presenza di metastasi in 123/140 casi con una sensibilità dell'87,9\% (Tabelle 4 e 5).

L'accuratezza complessiva tra esame ultrasonografico del cavo ascellare e istologico definitivo è risultata pari all' $85 \%$ (363/427), la sensibilità del 72,3\% (con il 27,7\% di falsi negativi ecografici) e la specificità del 93,4\%. L'accuratezza diagnostica tra FNAC del cavo ascellare e istologico definitivo è risultata del 93\% (120/129), la sensibilità dell'88,8\% e la specificità del 100\% (valori calcolati escludendo $i \mathrm{C1}$ ) (Tabella 6). Complessivamente l'ecografia associata alla FNAC ha modificato l'orientamento chirurgico del cavo ascellare in 71/147 donne (70 C5 e 1 C4, 48,3\%), che sono state sottoposte direttamente a dissezione del cavo ascellare evitando la biopsia del linfonodo sentinella.

I campioni inadeguati $(\mathrm{Cl})$ sono risultati 18 sul totale delle 147 pazienti sottoposte a FNAC (12,2\%). Nella maggior parte (12/18) l'agoaspirato è stato eseguito su linfonodi a basso sospetto (verosimilmente benigni); l'esame istologico definitivo ha confermato la benignità in 8/12 casi, mentre i restanti 4 sono risultati positivi per metastatizzazione. Negli altri 6 casi risultati Cl i linfonodi presentavano un aspetto ecografico fortemente suggestivo per malignità; non è stato ripetuto il prelievo per la loro sede profonda. Nella maggior parte di questi casi (4/6), l'istologico definitivo ha confermato la malignità ecografica, mentre solo 2 sono risultati non interessati da metastasi. 
Table 3 Concordance between axillary ultrasound (US), cytology and final histological examination

\begin{tabular}{|c|c|c|c|c|c|c|c|c|c|}
\hline \multirow[t]{2}{*}{ US appearance } & \multicolumn{5}{|c|}{ Cytology } & \multirow[t]{2}{*}{ Total } & \multicolumn{2}{|c|}{$\begin{array}{l}\text { Final histological } \\
\text { examination }\end{array}$} & \multirow[t]{2}{*}{ Concordance $(\%)$} \\
\hline & $\mathrm{C} 1$ & $\mathrm{C} 2$ & $\mathrm{C} 3$ & $\mathrm{C} 4$ & $\mathrm{C} 5$ & & Negative (N-) & Positive $(\mathrm{N}+)$ & \\
\hline Probably benign & 12 & 39 & 1 & 0 & 3 & 55 & 43 & 12 & 78.2 \\
\hline $\begin{array}{l}\text { Suspicious for malignancy } \\
\text { or probably malignant }\end{array}$ & 6 & 18 & 0 & 1 & 67 & 92 & 16 & 76 & 82.6 \\
\hline Total & 18 & 57 & 1 & 1 & 70 & 147 & 59 & 88 & \\
\hline
\end{tabular}

Tabella 3 Concordanza tra reperti ecografici linfonodali, citologia ed istologia definitiva

\begin{tabular}{|c|c|c|c|c|c|c|c|c|c|}
\hline \multirow[t]{2}{*}{ Aspetto ecografico } & \multicolumn{5}{|c|}{ Citologia } & \multirow[t]{2}{*}{ Totale } & \multicolumn{2}{|c|}{ Istologico definitivo } & \multirow[t]{2}{*}{ Concordanza (\%) } \\
\hline & $C 1$ & $C 2$ & C3 & $C 4$ & C5 & & Negativo $(N-)$ & Positivo $(\mathrm{N}+)$ & \\
\hline Verosimilmente benigno & 12 & 39 & 1 & 0 & 3 & 55 & 43 & 12 & 78,2 \\
\hline $\begin{array}{l}\text { Sospetto per malignità o } \\
\text { verosimilmente maligno }\end{array}$ & 6 & 18 & 0 & 1 & 67 & 92 & 16 & 76 & 82,6 \\
\hline Totale & 18 & 57 & 1 & 1 & 70 & 147 & 59 & 88 & \\
\hline
\end{tabular}

Table 4 Correlation between the longitudinal and transverse $(\mathrm{L} / \mathrm{T})$ ratio and final histological examination

\begin{tabular}{llll}
\hline L/T ratio & Total & \multicolumn{2}{c}{ Final histological examination } \\
& & Negative $(\mathrm{N}-)$ & Positive $(\mathrm{N}+)$ \\
\hline$\geq 2$ (normal) & 287 & 240 & 47 \\
$<2$ (pathological) & 140 & 17 & 123 \\
Total & 427 & 257 & 170 \\
\hline
\end{tabular}

Table 5 Accuracy of the longitudinal and transverse (L/T) ratio of lymph nodes

\begin{tabular}{llll}
\hline Sensitivity (\%) & Specificity (\%) & PPV (\%) & NPV (\%) \\
\hline 87.9 & 83.6 & 72.3 & 93.4 \\
\hline
\end{tabular}

$P P V$, positive predictive value; $N P V$, negative predictive value

Tabella 5 Accuratezza del rapporto L/T linfonodale

\begin{tabular}{llll}
\hline Sensibilità (\%) & Specificità $(\%)$ & $V P P(\%)$ & $V P N(\%)$ \\
\hline 87,9 & 83,6 & 72,3 & 93,4 \\
\hline
\end{tabular}

$V P P$, valore predittivo positivo; VPN, valore predittivo negativo

Overall, US combined with FNAC modified axillary treatment decisions in 71/147 women [(70 C5 and one C4) 48.3 $\%$ ] who proceeded directly to axillary dissection, thus avoiding sentinel node biopsy.

Inadequate samples $(\mathrm{C} 1)$ were seen in 18 out of a total of
Tabella 4 Relazione tra rapporto tra diametro longitudinale e trasversale (L/T) linfonodale e istologico definitivo

\begin{tabular}{llll}
\hline Rapporto L/T & Totale & \multicolumn{2}{c}{ Istologico definitivo } \\
& & Negativo $(N-)$ & Positivo $(N+)$ \\
\hline$\geq 2$ (normale) & 287 & 240 & 47 \\
$<2($ patologico $)$ & 140 & 17 & 123 \\
Totale & 427 & 257 & 70 \\
\hline
\end{tabular}

\section{Discussione}

L'ottimizzazione del percorso diagnostico nella stadiazione loco regionale del carcinoma della mammella costituisce un importante obiettivo della diagnostica senologica. In quest'ottica, l'impiego ormai consolidato, soprattutto per le lesioni infracliniche, di sistemi bioptici mini-invasivi, quali la vacuum assisted core biopsy (VACB), sia con guida stereotassica [29-31] e, sebbene più limitatamente, con guida RM [32, 33], stanno consentendo una più corretta pianificazione chirurgico-terapeutica, ampliando le possibilità in fase pre-operatoria dell'imaging senologico. Nel raggiungimento di una corretta stadiazione pre-operatoria del carcinoma mammario, non si può prescindere, tuttavia, dalla valutazione dell'interessamento metastatico linfonodale ascellare: l'eventuale diffusione alle stazioni linfonodali del cavo ascellare condiziona infatti fortemente la prognosi delle pazienti affette da tale neoplasia.

Nello studio ecografico del cavo ascellare, $i$ criteri più significativi per la presenza di metastasi linfonodali sono l'alterazione del rapporto tra $i$ diametri longitudinale $e$ 
Table 6 Diagnostic accuracy of axillary ultrasound (US) and fine-needle-aspiration cytology (FNAC) in identifying axillary metastases

\begin{tabular}{llllllllll}
\hline & TP & TN & FP & FN & Total & Sensitivity (\%) & Specificity (\%) & PPV (\%) & NPV (\%) \\
\hline Axillary US & 123 & 240 & 17 & 47 & 427 & 72.3 & 93.4 & 87.9 & 83.6 \\
FNAC $^{\text {a }}$ & 71 & 49 & 0 & 9 & 129 & 88.8 & 100 & 100 & 84.5 \\
\hline
\end{tabular}

$T P$, true positive; $T N$, true negative; $F P$, false positive; $F N$, false negative; $P P V$, positive predictive value; $N P V$, negative predictive value ${ }^{\mathrm{a}} \mathrm{C} 1$ excluded

Tabella 6 Accuratezza dell'ecografia e della citologia su agoaspirato per le metastasi ascellari

\begin{tabular}{lccccccccc}
\hline & $V P$ & $V N$ & $F P$ & $F N$ & Totale & Sensibilità $(\%)$ & Specificità (\%) & VPP (\%) & VPN (\%) \\
\hline $\begin{array}{l}\text { Ecografie } \\
\begin{array}{l}\text { ascellari } \\
\text { FNAC }^{a}\end{array}\end{array}$ & 123 & 240 & 17 & 47 & 427 & 72,3 & 93,4 & 87,9 & 83,6 \\
\hline
\end{tabular}

VP, veri positivi; VN, veri negativi; FP, falsi positivi; FN, falsi negatvi; VPP, valore predittivo positivo; VPN, valore predittivo negativo; FNAC, agoaspirato con ago sottile

${ }^{a}$ Cl esclusi

147 patients undergoing FNAC (12.2\%). In most cases (12/18) FNAC was performed on probably benign lymph nodes; the final histological examination confirmed benignity in 8/12 cases, whereas the remaining four were positive for metastasis. In the other six cases classified as C1, the lymph nodes exhibited a US appearance strongly suggestive of malignancy; sampling was not repeated due to deep location. In most of these cases (4/6), final histology confirmed the malignancy suspected at US, whereas only two were found to be free of metastases.

\section{Discussion}

Optimisation of the diagnostic workup in locoregional staging of breast carcinoma is an important goal of breast diagnostics. In this context, the consolidated use - especially in subclinical lesions - of minimally invasive biopsy systems such as vacuum-assisted core biopsy (VACB), whether stereotactically guided [29-31] or, more rarely, magnetic resonance imaging (MRI) guided [32, 33], are improving treatment planning by extending the possibilities of preoperative breast imaging. Correct preoperative staging of breast carcinoma, however, requires evaluation of axillary lymph node metastasis, as spread to the axillary nodes heavily impacts the prognosis of breast cancer patients.

In the US study of the axilla, the most significant criteria for detecting the presence of lymph node metastases are alteration of the ratio between longitudinal and transverse diameter, asymmetrical or focal cortical thickening and poor hilar visibility, as reported by numerous studies [1-26]. Other criteria, such as changes in size, are not sufficiently specific to distinguish between presence and absence of malignancy, given that lymph nodes may also be enlarged as a result of trasversale, l'ispessimento asimmetrico o focale della corticale e la mancata riconoscibilità della regione ilare, come già evidenziato in un significativo numero di lavori [1-26]. Altri parametri, quali le variazioni di dimensioni, non rappresentano invece un criterio specifico per la differenziazione fra la presenza o meno di patologia maligna. Infatti, i linfonodi possono essere ingranditi anche a seguito di processi infiammatori o di malattie sistemiche e, per contro, talvolta i linfonodi metastatici possono avere dimensioni inferiori al centimetro. I linfonodi di tipo reattivo, di frequente riscontro nel cavo ascellare, dal punto di vista ultrasonografico si presentano come strutture ovalari o reniformi di dimensioni generalmente superiori al centimetro (anche fino a 2,5 cm), con ilo iperecogeno ben evidente. Infatti, un prolungato stimolo patogeno può determinare un aumento numerico dei sinusoidi dei vasi linfatici del seno linfonodale, accentuando l'evidenza dell'ilo iperecogeno [2, 3].

A differenza di quanto accade nei linfonodi reattivi, nei linfonodi metastatici il processo interessa prevalentemente la sede sub-corticale e corticale in toto; perciò la corticale, invasa dal tessuto neoplastico, si ipertrofizza in modo non omogeneo, deformando o cancellando l'iperecogenicità dell'ilo e pertanto i sinusoidi linfatici [3]. L'ispessimento della corticale oltre i $2 \mathrm{~mm}$ (diffuso, focale o eccentrico), la progressiva alterazione ecostrutturale del linfonodo fino all'ipoecogenicità e l'ilo poco o per nulla visibile sono importanti criteri per la diagnosi di metastasi linfonodali.

Il rapporto fra diametro $L$ e $T$ del linfonodo tendente all'unità, definito roundness index da Sakai et al. [4], risulta in numerosi studi l'indice più attendibile di coinvolgimento linfonodale metastatico [4-8]. In accordo con quanto riportato in letteratura, anche nella nostra esperienza il rapporto $L / T$ (usando come soglia 2) ha dimostrato un elevato valore 
inflammatory processes or systemic disease, and metastatic lymph nodes may also be smaller than $1 \mathrm{~cm}$ in size. Reactive lymph nodes, a frequent finding in the axilla, appear on US as either oval or reniform structures usually larger than $1 \mathrm{~cm}$ (up to $2.5 \mathrm{~cm}$ ) and with a clearly visible hyperechoic hilum. In fact, a prolonged pathogenic process may lead to an increased number of lymphatic sinusoids, heightening the conspicuity of the hyperechoic hilum [2, 3].

Conversely, in metastatic lymph nodes, the process mainly involves the entire subcortical or cortical region, which, being invaded by the neoplastic tissue becomes inhomogeneously hypertrophic, deforming or cancelling the hyperechogenicity of the hilum and the lymphatic sinusoids [3]. Cortical thickening $>2 \mathrm{~mm}$ (diffuse, focal, eccentric), progressive echostructural changes in the lymph node up to hypoechogenicity and poorly visible or invisible hilum are all important criteria for the diagnosis of lymph node metastases

A ratio between $\mathrm{L}$ and $\mathrm{T}$ diameter of lymph nodes of approximately 1, defined as "roundness index" by Sakai et al. [4], has been found to be the most reliable index of nodal metastasis by a number of studies [5-8]. In agreement with the literature, we found the $\mathrm{L} / \mathrm{T}$ ratio (considering a value of 2 as the threshold) to have high NPV (93.4\%) and a lower PPV (72.3\%), probably because, as emphasised by Feu et al. [6] and Balu-Maestro et al. [9], very small benign lymph nodes with a longitudinal diameter $<1 \mathrm{~cm}$ are also nearly always round in shape. On the other hand, metastatic lymph nodes $>2 \mathrm{~cm}$ may maintain an oval shape.

In most published studies, lymph nodes are classified based on the above criteria into two categories only: normal or suspicious [2,7-26]. Considering all of the morphostructural alterations of axillary lymph nodes, in assessing the accuracy of US alone we proposed a classification of sonographic findings into four categories (normal, probably benign, suspicious for malignancy, probably malignant) in line with the Breast Imaging Reporting and Data System (BI-RADS) classification. By using this classification, concordance between US and final histology was $87.9 \%$ in probably malignant (Figs. 3) or suspicious (Fig. 2) lymph nodes, $75 \%$ in probably benign nodes (Fig. 1) and $87.2 \%$ in normal lymph nodes. Overall diagnostic accuracy of US was $85 \%$ and specificity was $93.4 \%$, whereas sensitivity was $72.3 \%$, in keeping with the literature that reports high specificity values $(80.4 \%-95.2 \%)$ and more variable sensitivity values $(64.3 \%-92.3 \%)$ [8, 10-16] (Table 7). In fact, in $27.7 \%$ of cases (47/170 false negative cases), negative axillary US findings were associated with positive axillary lymph nodes on final histology. The false negative rates of US reported by Nori et al. [7, 8] in 2005 and 2007 (27.3\% and $22.8 \%$, respectively) were comparable with those found in our study.

In our experience, the use of FNAC increased predittivo negativo (VPN=93,4\%) ed un valore predittivo positivo percentualmente inferiore (VPP=72,3\%), verosimilmente in base al fatto che, come sottolineato da Feu et al. [6] e Balu-Maestro et al. [9], i linfonodi benigni molto piccoli, con diametro longitudinale inferiore a $1 \mathrm{~cm}$, hanno anch'essi, quasi sempre, una forma rotondeggiante. Per contro, linfonodi metastatici maggiori di $2 \mathrm{~cm}$, possono mantenere una forma tendenzialmente ovale.

Nella maggior parte degli studi presenti in letteratura, $i$ linfonodi sono classificati in base ai criteri precedentemente descritti in due sole categorie: normali oppure sospetti [2, 7-26]. Considerando nell'insieme le alterazioni morfostrutturali dei linfonodi ascellari, abbiamo proposto nel nostro studio una classificazione dei reperti ecografici in quattro categorie (linfonodo normale, verosimilmente benigno, sospetto per malignità o verosimilmente maligno) in analogia con la classificazione breast imaging reporting and data system (BI-RADS), al fine di valutare l'accuratezza della sola ecografia. Utilizzando questa distinzione, abbiamo osservato una concordanza della sola ecografia con l'istologia definitiva rispettivamente pari all'87,9\% nei linfonodi verosimilmente maligni (Fig. 3) o sospetti per malignità (Fig. 2), al $75 \%$ nei verosimilmente benigni (Fig. 1) e all'87,2\% in quelli normali. Complessivamente, nella nostra esperienza l'accuratezza diagnostica dell'ecografia è risultata pari all' $85 \%$, la specificità 93,4\% e la sensibilità $72,3 \%$, in accordo con la letteratura, che riporta elevati valori di specificità $(80,4 \%-95,2 \%)$ e più variabili valori $d i$ sensibilità (64,3\%-92,3\%) (Tabella 7) [8, 10-16]. Infatti nel 27,7\% (47/170 casi di cosiddetti falsi negativi) alla negatività del quadro ultrasonografico dei cavi si è associata la positività dei linfonodi ascellari all'istologico definitivo. Anche nelle recenti casistiche (2005 e 2007) riportate da Nori et al. [7, 8], i falsi negativi all'esame ecografico (rispettivamente 27,3\% e 22,8\%) risultavano nel complesso sovrapponibili a quelli del nostro studio.

Nella nostra esperienza, l'impiego della FNAC ha incrementato in tutte le categorie la concordanza tra istologia definitiva e sospetto ecografico, in particolare nella categoria intermedia (aspetto del linfonodo verosimilmente benigno) rispettivamente dal $75 \%$ all' $88,4 \%$. Sono stati esaminati $i$ risultati della FNAC sotto guida ecografica della nostra casistica e confrontati con quelli più significativi della letteratura (Tabella 8) [1, 2, 14, 16-23]. La specificità è risultata elevata in tutti gli studi esaminati, oscillando dall' $89 \%$ al $100 \%$. Nella nostra esperienza, relativa a 147 FNAC, la specificità è stata del 100\%, poiché non ci sono stati falsi positivi.

La sensibilità complessiva documentata nella nostra serie è stata dell'88,8\% (valore calcolato escludendo i C1 dallo studio) ed è risultata molto soddisfacente in rapporto ai valori riportati in letteratura (variabili dal $57 \%$ al $94,9 \%)$, essendo inferiore solo a quella riportata in due 
Table 7 Sensitivity and specificity of axillary ultrasound (US): comparison with the literature data

\begin{tabular}{lll}
\hline Study (year) & Sensitivity $(95 \%$ CI) & Specificity (95\% CI) \\
\hline Vaidya et al. [10] & 69 & 89.7 \\
Lam et al. [11] & 72.7 & 95 \\
Verbanck et al. [12] & 92.3 & 95.2 \\
Yang et al. [13] & 79.5 & 95.2 \\
Sapino et al. [14] & 68.2 & 80.4 \\
Damera et al. [15] & 71.8 & 81.4 \\
Brancato et al. [16] & 64.3 & 92.8 \\
Nori et al. [8] & 72.7 & 90 \\
This study (2008) & 72.3 & 93.4 \\
\hline
\end{tabular}

CI, confidence interval

Tabella 7 Sensibilità e specificità della ecografia del cavo ascellare: confronto con i dati della letteratura

\begin{tabular}{lll}
\hline Autore dello studio (anno) & Sensibilità (IC 95\%) & Specificità (IC 95\%) \\
\hline Vaidya et al. [10] & 69 & 89,7 \\
Lam et al. [11] & 72,7 & 95 \\
Verbank et al. [12] & 92,3 & 95,2 \\
Yang et al. [13] & 79,5 & 95,2 \\
Sapino et al. [14] & 68,2 & 80,4 \\
Damera et al. [15] $[1,8$ & 81,4 \\
Brancato et al. [16] & 64,3 & 92,8 \\
Nori et al. [8] & 72,7 & 90 \\
Presente studio (2008) & 72,3 & 93,4 \\
\hline
\end{tabular}

IC, intervallo di confidenza

concordance between sonographic suspicion and final histology from $75 \%$ to $88.4 \%$ in all categories, and particularly in the intermediate category (probably benign appearance). The results of US-guided FNAC in our series were reviewed in comparison with the major published reports $[1,2,14$, 16-23] (Table 8). All studies reported a high level of specificity, ranging from $89 \%$ to $100 \%$. In our experience with 147 FNAC, specificity reached $100 \%$, with no false positive cases.

Overall sensitivity in our series was $88.8 \%$ (a value calculated excluding $\mathrm{C} 1$ cases from the analysis). This value was considered highly satisfactory when compared with previous reports (ranging from 57\% to 94.9\%). In fact, although lower than that reported by Duchesne et al. and De Kanter et al. [1, 17], who performed significantly fewer FNAC procedures (83 and 69, respectively), it was higher than reported in nine other papers [2, 14, 16, 18-23]. In the studies of Bonnema et al. [18] and Kuenen-Boumeester et al. [19], who analysed 81 and 183 patients subjected to FNAC, sensitivity was significantly lower (63\% and $57 \%$, respectively), as the authors considered subclinical lymph nodes only.

The major limitation of FNAC is, however, the number of inadequate or nondiagnostic samples. This reached $12.2 \%$ in our series compared with a reported rate of $8.2 \%-30 \%[16,22,23]$. More specifically, the majority of altre casistiche [1, 17], con un numero di agoaspirati linfonodali decisamente inferiore (rispettivamente 69 e 83), mentre è parsa più elevata rispetto agli altri 9 lavori esaminati [1, 2, 14, 16-23]. Nelle casistiche di Bonnema et al. [18] e di Kuenen-Boumeester et al. [19], relative a 81 e 183 pazienti sottoposte a FNAC, la sensibilità è risultata decisamente più bassa (rispettivamente del 63\% e 57\%) poiché sono stati considerati unicamente i linfonodi infraclinici.

Tuttavia, il maggior limite della FNAC è costituito dal numero di campioni inadeguati o non diagnostici, che in questo lavoro sono risultati pari al 12,2\%; in letteratura tale percentuale risulta compresa tra l'8,2\% e il 30\% [16, 22, 23]. In particolare, abbiamo osservato che il maggior numero di campioni inadeguati è stato riscontrato nei prelievi su linfonodi ad aspetto verosimilmente benigno (indeterminato) (12/18). Per ovviare a questo limite, in alcuni recenti lavori è stato proposto l'utilizzo della core biopsy: con questa metodica è stato riportato un incremento della sensibilità sino al 94\% [7, 8, 15, 24-26]. Tuttavia, tale procedura non viene utilizzata su larga scala per la stadiazione dei linfonodi ascellari soprattutto perché è più invasiva rispetto all'agoaspirato linfonodale; la difficoltà principale nell'esecuzione della core biopsy consiste nel riuscire ad evitare di danneggiare $i$ vasi sanguigni o i nervi, poiché $i$ linfonodi sono spesso localizzati in prossimità di queste strutture [25, 26]. Pur con le limitazioni sia della metodica 
Table 8 Sensitivity and specificity of combined axillary ultrasound (US) and fine-needle-aspiration cytology (FNAC): comparison with the literature data

\begin{tabular}{llll}
\hline Study (year) & Sensitivity (95\% CI) & Specificity (95\% CI) & No. of US-FNAC procedures \\
\hline Bonnema et al. [18] & 79.5 & 100 & 81 \\
De Kanter et al. [17] & 94.9 & 100 & 69 \\
Krishnamurthy et al. [2] & 84.8 & 100 & 103 \\
Kuenen-Boumeester et al. [19] & 57 & 96.9 & 183 \\
Deurloo et al. [20] & 75.5 & 100 & 66 \\
Sapino et al. [14] & 81.6 & 100 & 95 \\
Brancato et al. [16] & 58.6 & 100 & 159 \\
Duchesne et al. [1] & 94 & 89 & 83 \\
Podkrajsek et al. [21] & 84 & 91 & 49 \\
Ciatto et al. [22] & 72.6 & 95.7 & 491 \\
Altomare et al. [23] & 68 & 100 & 100 \\
This study (2008) & 88.8 & 100 & 147 \\
\hline
\end{tabular}

CI, confidence interval

Tabella 8 Sensibilità e specificità dell'agoaspirato con ago sottile ecoguidato (US-FNAC) ascellare: confronto con i dati della letteraturaù

\begin{tabular}{llll}
\hline Autore dello studio (anno) & Sensibilità (IC 95\%) & Specificità (IC 95\%) & Numero di US-FNAC \\
\hline Bonnema et al. [18] & 79,5 & 100 & 81 \\
De Kanter et al. [17] & 94,9 & 100 & 69 \\
Krishnamurthy et al. [2] & 84,8 & 100 & 103 \\
Kuenen-Boumeester et al. [19] & 57 & 96,9 & 183 \\
Deurloo et al. [20] & 75,5 & 100 & 66 \\
Sapino et al. [14] & 81,6 & 100 & 95 \\
Brancato et al. [16] $[1]$ & 58,6 & 100 & 159 \\
Duchesne et al. [1] & 94 & 89 & 83 \\
Podkarajsek et al. [21] & 84 & 91 & 49 \\
Ciatto et al. [22] & 72,6 & 95,7 & 491 \\
Altomare et al. [23] & 68 & 100 & 100 \\
Presente studio (2008) & 88,8 & 100 & 147 \\
\hline
\end{tabular}

IC, intervallo di confidenza

inadequate samples concerned lymph nodes with probably benign (indeterminate) appearance (12/18). To overcome this limitation, recent studies have proposed the use of core biopsy, a method that been shown to increase sensitivity to $94 \%[7,8,15,24-26]$. However, core biopsy is not widely used for axillary lymph-node staging owing to its greater aggressiveness compared with FNAC. The main difficulty in performing core biopsy lies in avoiding damage to blood vessels or nerves, given that lymph nodes are often located in the vicinity of these structures $[25,26]$. Despite the limitations of both US and needle biopsy, combined US and FNAC in our study allowed sentinel node biopsy to be avoided in almost half of the patients $(71 / 147 ; 48.3 \%)$, who were thus referred for immediate axillary dissection.

Our results suggest that in women with proven breast carcinoma, it may be appropriate to characterise lymph nodes with FNAC whenever nodal abnormalities are detected on US, even those with probably benign appearance. This is because the goal of lymph node characterisation is not to rule out metastatic involvement but rather to search for signs of secondary disease to avoid two-step axillary surgery. ecografica, sia della metodica agobioptica, nel nostro studio l'ecografia associata a FNAC ha infatti permesso di evitare la biopsia del linfonodo sentinella in quasi la metà delle pazienti $(71 / 147,48,3 \%)$ orientando il trattamento chirurgico verso una dissezione ascellare immediata.

Questi risultati suggeriscono che nelle donne con diagnosi accertata di neoplasia mammaria, sembrerebbe opportuno ricorrere alla caratterizzazione dei linfonodi mediante FNAC ogni qual volta si riscontrino alterazioni linfonodali, anche se più verosimilmente orientate verso la benignità ecostrutturale. Infatti, l'obiettivo dello studio linfonodale non è l'esclusione dell'interessamento metastatico, bensì la ricerca dell'eventuale positività per secondarietà, al fine di evitare la chirurgia del cavo ascellare in due tempi.

\section{Conclusioni}

L'ecografia dei linfonodi del cavo ascellare è un esame rapido, non invasivo, per cui dovrebbe essere eseguita di routine nelle pazienti affette da neoplasia mammaria, potendo svolgere un importante ruolo nella valutazione 


\section{Conclusions}

Axillary US is a fast, noninvasive examination that should be performed routinely in patients with breast carcinoma, as it can play a major role in the preoperative evaluation of metastatic spread. The main limitation of axillary US is its high false negative rate resulting from the presence of micrometastases in normal-appearing nodes and the failure to visualise all lymph nodes.

On the basis of our experience, the preoperative staging of breast cancer patients scheduled for surgery can be improved by combining the US study of suspicious axillary nodes and US-guided FNAC. In our series, axillary US combined with FNAC led to a preoperative diagnosis of nodal metastasis in almost half of the patients (48.3\%), who proceeded to immediate axillary dissection with a substantial reduction of costs, length of hospital stay and patients' psychological distress. pre-operatoria nell'eventualità di un coinvolgimento metastatico. Il limite dell'ecografia del cavo ascellare è rappresentato dal considerevole numero di falsi negativi, in parte dovuti alla presenza di micrometastasi in linfonodi con una struttura ecografica apparentemente normale ed in parte all'impossibilità di riconoscere tutti $i$ linfonodi durante l'indagine ecografica.

In base alla nostra esperienza, se la valutazione ecografica di linfonodi sospetti del cavo ascellare viene associata alla citologia ecoguidata (FNAC) è possibile migliorare la stadiazione pre-operatoria delle pazienti affette da neoplasia mammaria candidate a trattamento chirurgico. Nella casistica in esame, l'ecografia del cavo ascellare associata a FNAC ha infatti permesso una diagnosi preoperatoria di metastasi linfonodali in circa la metà delle pazienti $(48,3 \%)$, orientando verso una dissezione ascellare immediata, con sensibile riduzione dei costi, del tempo di ospedalizzazione e del disagio psicologico delle pazienti.

Conflict of interest statement The authors declare that they have no conflict of interest to the publication of this article.

\section{References/Bibliografia}

1. Duchesne N, Jaffey J, Florack P et al (2005) Redefining ultrasound appearance criteria of positive axillary lymph nodes. Can Assoc Radiol J 56:289-296

2. Krishnamurthy S, Sneige N, Bedi DG et al (2002) Role of ultrasound-guided fine needle aspiration of indeterminate and suspicious axillary lymph nodes in the initial staging of breast carcinoma. Cancer 95:982-988

3. Stavros AT (2004) Evaluation of regional lymph nodes in breast cancer patients. In: Stavros AT (ed) Breast ultrasound. Lippincott Williams and Wilkins, Philadelphia, pp 834-876

4. Sakai F, Kiyono K, Sone S et al (1998) Ultrasonic evaluation of cervical metastatic lymphadenopathy. J Ultrasound Med 7:305-310

5. Vassallo P, Wernecke K, Roos N et al (1992) Differentiation of benign from malignant superficial lymphadenectomy. The role of highresolution US. Radiology 183:215-220

6. Feu J, Tresserra F, Fabregas R et al (1997) Metastatic breast carcinoma in axillary lymph-nodes: in vitro US detection. Radiology 205:831-835

7. Nori J, Vanzi E, Bazzocchi M et al (2007) Role of axillary ultrasound examination in the selection of breast cancer patients for sentinel node biopsy. Am J Surg 193:16-20
8. Nori J, Bazzocchi M, Boeri C et al (2005) Ruolo dell'ecografia e della microbiopsia istologica nella valutazione del cavo ascellare in pazienti candidate a biopsia del linfonodo sentinella. Radiol Med 109:330-344

9. Balu Maestro C, Chapellier C, Carrier $P$ et al (2005) Role of imaging in the evaluation of axillary nodes and metastatic disease from breast carcinoma. J Radiol 86:1649-1657

10. Vaidya JS, Vyas JJ, Thakur MH et al (1996) Role of ultrasonography to detect axillary node involvement in operable breast cancer. Eur J Surg Oncol 22:140-143

11. Lam WW, Yang WT, Chan YL et al (1996) Detection of axillary lymph node metastases in breast carcinoma by technetium-99m sestamibi breast scintigraphy, ultrasound and conventional mammography. Eur J Nucl Med 23:498-503

12. Verbanck J, Vandewiele I, De Winter $\mathrm{H}$ et al (1997) Value of axillary ultrasonography and sonographically guided puncture of axillary nodes: a prospective study in 144 consecutive patients. J Clin Ultrasound 25:53-56

13. Yang WT, Ahuja A, Tang A et al (1995) Ultrasonographic demonstration of normal axillary lymph nodes: a learning curve. J Ultrasound Med 14:823-827
14. Sapino A, Cassoni P, Zanon E et al (2003) Ultrasonographically-guided fine-needle aspiration of axillary lymph nodes: role in breast cancer management. Br J Cancer 88:702-706

15. Damera A, Evans AJ, Cornford et al (2003) Diagnosis of axillary nodal metastases by ultrasound-guided core biopsy in primary operable breast cancer. Br J Cancer 89:1310-1313

16. Brancato B, Zappa M, Bricolo D et al (2004) Role of ultrasound-guided fine needle cytology of axillary lymph nodes in breast carcinoma staging. Radiol Med 108:345-355

17. De Kanter AY, van Eijck CHJ, van Geel AN et al (1999) Multicentre study of ultrasonographically guided axillary node biopsy in patients with breast cancer. Br J Surg 86:1459-1462

18. Bonnema J, van Geel AN, van Ooijen B et al (1997) Ultrasound guided aspiration biopsy for detection of non palpable axillary node metastases in breast cancer patients: new diagnostic method. World J Surg 21:270-274

19. Kuenen-Boumeester V, MenkePluymers M, de Kanter AY et al (2003) Ultrasound-guided fine needle aspiration cytology of axillary lymph nodes in breast cancer patients: a preoperative staging procedure. Eur J Cancer 39:170-174 
20. Deurloo EE, Tanis PJ, Gilhuijs KG et al (2003) Reduction in the number of sentinel lymph node procedures by preoperative ultrasonography of the axilla in breast cancer. Eur J Cancer 39:1068-1073

21. Podkrajsek M, Music MM, Kadivec M et al (2005) Role of ultrasound in the preoperative staging of patients with breast cancer. Eur Radiol 15:1044-1050

22. Ciatto $S$, Brancato B, Risso $G$ et al (2007) Accuracy of fine needle aspiration cytology (FNAC) of axillary lymph nodes as a triage test in breast cancer staging. Breast Cancer Res Treat 103:85-91

23. Altomare V, Guerriero G, Carino R et al (2007) Axillary lymph node echoguided fine-needle aspiration cytology enables breast cancer patients to avoid a sentinel lymph node biopsy.

Preliminary experience and a review of the literature. Surg Today 37:735-739
24. Topal U, Punar S, Tasdelen I et al (2005) Role of ultrasound-guided core needle biopsy of axillary lymph nodes in the initial staging of breast carcinoma. Eur J Radiol 56:382-385

25. Abe H, Schmidt RA, Sennett CA et al (2007) US-guided core needle biopsy of axillary lymph nodes in patients with breast cancer: why and how to do it. Radiographics 27:S91-S99

26. Abe H, Schmidt RA, Kulkarni K et al (2008) Axillary lymph nodes suspicious for breast cancer metastasis: sampling with US-guided 14 gauge core-needle biopsy - Clinical experience in 100 patients. Radiology 250:41-49

27. Perry N, Broeders M, de Wolf C et al (2006) European Guidelines for Quality Assurance in Breast Cancer Screening and Diagnosis (4th edition). Office for Official Publications of the European Communities, Luxembourg

28. Elston CW, Ellis IO (1991) Pathological prognostic factors in breast cancer. I. The value of histological grade in breast cancer: experience from a large study with long-term follow-up. Histopathol 19:403-410
29. Zuiani C, Mazzarella F, Londero V et al (2007) Stereotactic vacuum-assisted breast biopsy: results, follow up and correlation with radiological suspicion. Radiol Med 112:304-317

30. Tonegutti M, Girardi V (2008) Stereotactic vacuum assisted breast biopsy in 268 nonpalpable lesions. Radiol Med 113:65-75

31. Jackman RJ, Marzoni FA Jr, Rosenberg J (2009) False negative diagnoses at stereotactic vacuum assisted needle breast biopsy: long term follw up of 1280 lesions and review of the literature. AJR Am J Roentgenol 192:341-351

32. Gebauer B, Bostanjoglo M, Moesta KT et al (2006) Magnetic resonance guided biopsy of suspicious breast lesions with a handheld vacuum biopsy device. Acta Radiol 47:907-913

33. Perretta T, Pistolese CA, Bolacchi F et al (2008) MR imaging-guided 10-gauge vacuum-assisted breast biopsy: histological characterisation. Radiol Med 113:830-840 